\title{
QUALIDADE DE SERVIÇOS EM SAÚDE: UMA AVALIAÇÃO EM AMBULATÓRIOS GERAIS DE UMA CIDADE LOCALIZADA NO SUL DO BRASIL.
}

Sheila Patrícia Ramos Beckhauser ${ }^{1}$

Gerson Tontini ${ }^{2}$

Patric Douglas Grisele ${ }^{3}$

Anemir Maria Kerber Ciotti ${ }^{4}$

Rodrigo Campos Ferreira ${ }^{5}$

\section{Resumo}

O presente estudo tem como objetivo demostrar como avaliar a qualidade de serviços em saúde pública, e decidir o que melhorar. Para tanto, adotou-se uma metodologia quantitativa quanto à abordagem de pesquisa, levantamento quanto aos procedimentos, e exploratória quanto aos objetivos. A população do estudo foi composta por usuários dos serviços público de saúde, especificamente Ambulatórios Gerais, na cidade de Blumenau/SC. A amostra foi de 355 respondentes. Investigou-se o impacto de oito dimensões na avaliação geral dos usuários sobre os serviços prestados. Utilizando a análise da lacuna de importância - desempenho para identificação de prioridades de melhoria, concluiu-se a seguinte ordem de prioridade de esforços para melhoria dos serviços: 1 - Tempo para atendimento; 2 - Farmácia; 3 - Serviços Disponíveis; 4 - Atendimento; 5 - Comunicação; 6 - Confiança; 7 - Instalações; 8 - Acesso aos ambulatórios. Conclui-se que, embora os usuários do serviço de saúde pública se encontrem satisfeitos, ações podem ser realizadas para que sejam melhorados. Ademais, o presente artigo mostra como a análise de importância x desempenho pode colaborar à decisão de ações de gestão de hospitais.

Palavras-chave: Satisfação. Qualidade. Serviços de Saúde Pública. Análise de Importância e Desempenho.

\begin{abstract}
The present study aims to demonstrate how to evaluate the quality of public health services, and decide what to improve. For that, a quantitative methodology was adopted regarding the research approach, survey on the procedures, and exploratory regarding the objectives. The study population consisted of users of public health services, specifically Ambulatorios Gerais, in the city of Blumenau / SC. The sample consisted of 355 respondents. We investigated the impact of eight dimensions on the general evaluation of users about the services provided. Using analysis of the gap of importance - performance to identify improvement priorities, the following order of priority of efforts to improve services was concluded: 1 - Time to attend; 2 - Pharmacy; 3 - Available Services; 4 - Attendance; 5 - Communication; 6 - Trust; 7 Installations; 8 - Access to outpatient clinics. We conclude that although public health service users are satisfied, actions can be taken to improve them. In addition, the present article shows how the analysis of importance $\mathrm{x}$ performance can contribute to the decision of actions of hospital management.
\end{abstract}

Keywords: Satisfaction. Quality. Public Health Services. Analysis of Importance and Performance.

\footnotetext{
${ }^{1}$ Mestre em Administração pela Universidade Regional de Blumenau (FURB). Doutoranda no Programa de PósGraduação em Ciências Contábeis e Administração (PPGCCA) da Universidade Regional de Blumenau (FURB).

2 Doutor pela Universidade Federal de Santa Catarina. Professor do Programa de Pós-Graduação em Ciências Contábeis e Administração (PPGCCA) da Universidade Regional de Blumenau (FURB).

${ }^{3}$ Mestre em Administração pela Universidade Regional de Blumenau (FURB).

${ }^{4}$ Mestrando em Administração pela Universidade Regional de Blumenau (FURB).

${ }^{5}$ Mestrando em Administração pela Universidade Regional de Blumenau (FURB).
} 


\section{INTRODUÇÃO}

Fatores de concorrência no mercado conduzem às empresas na busca pelo entendimento daquilo que influencia na satisfação do consumidor em relação aos serviços que dispõem. Obter dados que refletem este relacionamento fornece subsídios no desenvolvimento de estratégias de melhoria ou manutenção dos serviços oferecidos (MALIK, 2012).

Uma das principais características dos serviços é a sua intangibilidade. Serviços são avaliados pelo desempenho e experiência de quem os utiliza, tendo variabilidade em relação à percepção de cada pessoa (PENA et al., 2013). A qualidade dos serviços possui relação com a expectativa gerada pelo cliente e a sua satisfação com a experiência dos consumidores (MALIK, 2012).

$\mathrm{Na}$ área da saúde, os gestores dirigem maiores esforços em relação à garantia da segurança do paciente, na redução dos custos médicos e hospitalar, no serviço adequado ao paciente e na minimização de erros médicos. Também trabalham na construção de um sistema com equipamentos de qualidade, funcionários capacitados com incentivos que direcionam a um melhor desempenho organizacional (LEE; LEE; KANG, 2012).

Na prestação de serviços na área de saúde pesquisas de satisfação são amplamente utilizadas como ferramentas de gestão tendo grande relevância nas decisões estratégicas. A incorporação da avaliação como prática sistemática nos diversos níveis dos serviços de saúde poderá propiciar aos seus gestores as informações requeridas para a definição de estratégias de intervenção (SILVA; FORMIGLI, 1994).

Pesquisas de satisfação dos usuários são indispensáveis ao planejamento e à avaliação dos serviços de saúde, pois, quanto mais as políticas públicas de saúde estiverem justificadas pelo respeito às perspectivas e às necessidades dos usuários, mais estas políticas tenderão ao acerto (FRÉZ et al., 2011).

Deste modo, o presente estudo tem como objetivo avaliar a qualidade dos serviços em saúde pública na cidade de Blumenau. Com isso busca-se, além de atender aos interesses da população ou usuários, pretende-se contribuir para futuras estratégias que podem ser aplicadas por meio do setor público responsável, e assim melhorar a qualidade dos serviços em saúde.

$\mathrm{O}$ artigo segue estruturado em mais quatro seções: na segunda seção apresentam-se a revisão bibliográfica, em que se aborda o tópico qualidade em serviços, qualidade dos serviços em saúde e Análise de Importância e Desempenho; na terceira seção, descrevem-se os procedimentos metodológicos; na quarta, destaca-se a apresentação e análise dos resultados e na quinta seção apresentam-se as considerações finais do estudo.

\section{REFERENCIAL TEÓRICO}

\subsection{Qualidade em serviços}

A qualidade dos serviços pode ser percebida pelos clientes por meio dos seus componentes tangíveis e intangíveis. A tangibilidade de um serviço é aquilo que o cliente vê e sente, como a aparência física do local de trabalho. Os componentes intangíveis são amabilidade e cordialidade da equipe de odontologia, ou seja, os que estão diretamente ligados ao relacionamento da equipe profissional com os clientes (ANSUJ; ZENCKNER; GODOY, 2005).

Estudos de satisfação podem contribuir para a institucionalização da avaliação e reorganização dos serviços além de ser uma ferramenta para a participação popular. A 
satisfação é um processo dinâmico que pode ser influenciado por uma série de fatores como percepção do estado de saúde e da doença, crenças, características sócio demográficas e envolve diversos aspectos de atenção (GIOVANELLA et al. 2013).

Segundo Esperidião e Trad (2006) a despeito das dificuldades em definir a satisfação, muitas pesquisas buscaram estudar a relação entre a satisfação e as variáveis que a determinam. Apesar do esforço dessas investigações, ainda se sabe pouco sobre a natureza ou o número de fatores que influenciam a satisfação. Esperidião e Trad (2006) afirmam que os resultados encontrados na literatura são divergentes, indicando baixa consistência das pesquisas.

Grönroos (1984) desenvolveu um dos primeiros modelos para mensuração da qualidade em serviços. Mais tarde, Parasuraman et al., (1985) propuseram a medição de qualidade do serviço, baseado em um modelo de satisfação. Que mais tarde foi complementado e denominado Modelo SERVQUAL.

Para Parasuraman, Zeithaml e Berry $(1985,1988)$ a qualidade em serviços representa a avaliação geral dos clientes, no que tange ao desempenho organizacional que pode ser estimada a partir das dez dimensões definidas por: confiabilidade, presteza, competência, acessibilidade, cortesia, comunicação credibilidade, segurança, compreensão e conhecimento do cliente e aspectos tangíveis. Posteriormente reduzidas a cinco dimensões: confiabilidade, presteza, segurança, empatia e aspectos tangíveis.

Além de Grönroos (1984) e do Modelo SERVQUAL de Parasuraman et al. $(1985,1988)$, surgiram outros modelos como: Modelo de avaliação de serviço e valor de Bolton e Drew (1991); Modelo SERVPERF de Cronin e Taylor (1992), Modelo de desempenho ideal de Teas (1993), entre outros.

\subsection{Qualidade dos serviços em saúde}

Vaitsman et al. (2005) afirmam que as primeiras pesquisas no campo da avaliação em saúde, ainda em 1970, referiam-se à satisfação do paciente. O objetivo era conseguir melhores resultados clínicos, por meio da adesão ao tratamento, em três dimensões: comparecimento às consultas; aceitação das recomendações e prescrições e uso adequado dos medicamentos. Aos poucos, quando satisfação do usuário passou a denominar um conjunto amplo e heterogêneo de pesquisas, com o objetivo de saber a opinião dos usuários de serviços de um modo geral, públicos ou privados, o termo usuário também passa a ser utilizado nas pesquisas de avaliação em saúde. Essas pesquisas vieram focalizar as distintas dimensões que envolvem o cuidado à saúde, desde a relação médico- paciente até a qualidade das instalações do serviço, passando pela qualidade técnica dos profissionais de saúde.

De acordo com Esperidião e Trad (2006) o sentido ao se teorizar no campo da avaliação dos serviços de saúde é, sobretudo, o de buscar converter os conceitos em estratégias, critérios e padrões de medição, a fim de contribuir para a produção de medidas úteis que auxiliem na tomada de decisão e subsidiem aperfeiçoamentos no âmbito dos serviços. A utilização dos conceitos se torna importante na medida em que permitem representar aspectos da realidade, através de construções lógicas estabelecidas de acordo com um quadro teórico de referência.

Existem várias razões pelas quais a pesquisa com usuários de serviços de saúde podem ser considerados úteis, incluindo: compreender os problemas atuais na prestação de cuidados; indicativos para melhoria contínua e redesenho de serviços; ajudar os profissionais refletir sobre sua própria prática e da sua equipe; acompanhar o impacto de quaisquer alterações; facilitar a análise comparativa dos serviços/organizações; avaliação de desempenho; Informar os profissionais sobre a qualidade dos serviços que prestam; permitir responsabilidade pública (COULTER; FITZPATRICK; CORNWELL, 2009). 
Segundo Dinsdale et al (2000), é preciso levar em conta algumas diferenças relevantes quando se avaliam serviços públicos e privados. No setor público, o usuário pode se colocar ora como consumidor, avaliando os serviços do ponto de vista de seus ganhos individuais, ora como cidadão, avaliando os serviços ao levar em conta a sociedade como um todo. Como consumidor, ele pode desejar um tempo de espera menor para a consulta e atendimento mais ágil, mas, como cidadão, pode querer que todas as pessoas sejam atendidas, implicando um tempo de espera maior. No que se refere à escolha, no setor privado, o cliente pode procurar outro serviço ou outro profissional, enquanto no setor público essa possibilidade é limitada, quando não impossível.

Vaitsman et al (2005) destacam que, no setor público, a avaliação feita a partir dos usuários envolve uma interação mais complexa de elementos. Por exemplo, o sentimento de gratidão pode dificultar uma visão mais crítica do atendimento. Os pacientes evitariam criticar os serviços devido a este viés, tanto pelo medo de perder o acesso, quanto à relação de dependência aos profissionais de saúde. Além disso, diferentes expectativas, seja entre indivíduos de distintos países ou mesmo dentro de um mesmo país que comporte distintas realidades socioculturais, introduzem, portanto, um viés subjetivo, ainda que as metodologias mais comuns de pesquisa de satisfação apresentem seus resultados de forma agregada e quantificada. Giovanella et al (2013) também destacam que a satisfação é um processo dinâmico que pode ser influenciado por uma série de fatores como a percepção do estado de saúde e da doença, crenças, características sócio demográficas e envolve diversos aspectos de atenção.

Os estudos relativos a avaliação de serviços em saúde destacam que: a satisfação de serviços de saúde na área pública (FRÉZ et al., 2011); a qualidade é percebida por meio do serviço interpessoal prestado e como os usuários são tratados pelos profissionais (FADEL; REGIS FILHO, 2009); que existem queixas quanto ao atendimento, falta de humanização e acolhimento, deficiência de recursos físicos e materiais (MOIMAZ et al, 2010); e que instrumentos de avaliação se mostram como uma ferramenta relevante na avaliação dos serviços (GIOVANELLA et al, 2013).

Destaca-se, na Tabela 1, estudos e algumas dimensões que foram avaliadas em serviços de saúde.

Tabela 1: Estudos em serviços de saúde.

\begin{tabular}{|c|c|c|c|}
\hline Autores & Dimensões & Objetivos & Resultados \\
\hline $\begin{array}{l}\text { Soufi et al } \\
(2010)\end{array}$ & $\begin{array}{l}\text { Atendimento } \\
\text { Tempo } \\
\text { Segurança/confiabilidade } \\
\text { no serviço }\end{array}$ & $\begin{array}{l}\text { Confirmar a confiabilidade e } \\
\text { validade da versão árabe do } \\
\text { EQS-H (Echelle de Qualité des } \\
\text { Soins em Hospitalisation) e } \\
\text { avaliar a satisfação dos } \\
\text { pacientes em um departamento } \\
\text { de medicina. }\end{array}$ & $\begin{array}{l}\text { A versão árabe do EQS-H } \\
\text { demonstrou } \\
\text { consistência. A maioria dos } \\
\text { pacientes ficaram satisfeitos com o } \\
\text { atendimento. }\end{array}$ \\
\hline $\begin{array}{l}\text { Barbosa } \\
(2009)\end{array}$ & $\begin{array}{l}\text { Atendimento; } \\
\text { Instalações; } \\
\text { Acesso; } \\
\text { Tempo atendimento; } \\
\text { Disponibilidade de } \\
\text { serviço; } \\
\text { Farmácia e } \\
\text { medicamentos; } \\
\text { Segurança; }\end{array}$ & $\begin{array}{l}\text { Construir uma metodologia de } \\
\text { avaliação de desempenho e } \\
\text { resultados de equipes da Saúde } \\
\text { de Família }\end{array}$ & $\begin{array}{l}\text { Os resultados evidenciaram uma } \\
\text { lacuna no que se refere à } \\
\text { realização do processo de trabalho } \\
\text { e um déficit de infraestrutura } \\
\text { disponível para dar suporte às } \\
\text { atividades das equipes, o que deixa } \\
\text { clara a necessidade de atuação das } \\
\text { esferas cabíveis na Estratégia de } \\
\text { Saúde da Família para que se }\end{array}$ \\
\hline
\end{tabular}




\begin{tabular}{|c|c|c|c|}
\hline & $\begin{array}{l}\text { Confiabilidade no } \\
\text { serviço }\end{array}$ & & $\begin{array}{l}\text { possa, de fato, inverter o modelo } \\
\text { de saúde tradicional. }\end{array}$ \\
\hline $\begin{array}{l}\text { Fadel e } \\
\text { Regis } \\
\text { Filho } \\
\text { (2009) }\end{array}$ & $\begin{array}{l}\text { Atendimento } \\
\text { Comunicação } \\
\text { Instalações } \\
\text { Acesso } \\
\text { Tempo atendimento }\end{array}$ & $\begin{array}{l}\text { Investigar a percepção de } \\
\text { clientes e profissionais } \\
\text { referente a qualidade de um } \\
\text { serviço público de odontologia }\end{array}$ & $\begin{array}{l}\text { Verificou-se diferença de } \\
\text { percepção entre os profissionais e } \\
\text { os clientes. Os profissionais } \\
\text { consideram de grande relevância a } \\
\text { qualidade técnica dos serviços e os } \\
\text { clientes consideram as qualidades } \\
\text { interpessoais, quais sejam o } \\
\text { cuidado e o tratamento pelos } \\
\text { profissionais. }\end{array}$ \\
\hline $\begin{array}{l}\text { Cho et al } \\
(2004)\end{array}$ & $\begin{array}{l}\text { Atendimento } \\
\text { Instalações } \\
\text { Tempo atendimento }\end{array}$ & $\begin{array}{l}\text { Examinar o impacto da } \\
\text { percepção dos clientes } \\
\text { externos na qualidade em } \\
\text { serviços hospitalares e testar a } \\
\text { invariância das relações } \\
\text { estruturais entre as dimensões } \\
\text { da qualidade de serviço e } \\
\text { satisfação. }\end{array}$ & $\begin{array}{l}\text { Verificou-se uma relação causal } \\
\text { entre a qualidade de serviço e } \\
\text { satisfação, bem como as } \\
\text { dimensões de qualidade de serviço } \\
\text { mostraram variação de padrões na } \\
\text { satisfação dos pacientes. }\end{array}$ \\
\hline $\begin{array}{l}\text { Coulter, } \\
\text { Fitzpatrick } \\
\text { e Cornwell } \\
(2009)\end{array}$ & $\begin{array}{l}\text { Atendimento } \\
\text { Instalações } \\
\text { Tempo atendimento } \\
\text { Segurança } \\
\text { Confiabilidade no } \\
\text { serviço }\end{array}$ & $\begin{array}{l}\text { Fornecer um breve guia para os } \\
\text { vários métodos que medem a } \\
\text { experiência dos pacientes nos } \\
\text { hospitais para ajudar as partes } \\
\text { interessadas a decidir quais } \\
\text { ferramentas de medição e } \\
\text { feedback são apropriados para } \\
\text { as suas necessidades. }\end{array}$ & 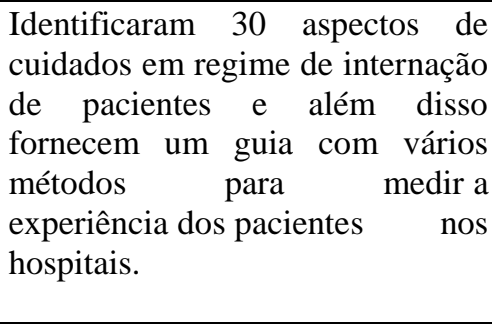 \\
\hline $\begin{array}{l}\text { Milan e } \\
\text { Trez } \\
(2005)\end{array}$ & $\begin{array}{l}\text { Atendimento } \\
\text { Instalações } \\
\text { Acesso } \\
\text { Tempo atendimento } \\
\text { Farmácia/medicamentos }\end{array}$ & $\begin{array}{l}\text { Identificar as dimensões de } \\
\text { análise que compõem a } \\
\text { avaliação da satisfação por } \\
\text { parte dos usuários dos serviços } \\
\text { de saúde desenvolver um } \\
\text { modelo de avaliação da } \\
\text { satisfação de clientes para } \\
\text { empresas de medicina de grupo } \\
\text { no Brasil. }\end{array}$ & $\begin{array}{l}\text { Foram identificadas dimensões de } \\
\text { avaliação dos serviços, quais } \\
\text { sejam o atendimento prestado, } \\
\text { estrutura do plano de saúde, } \\
\text { médicos, preço dos serviços, } \\
\text { comunicação do plano e } \\
\text { conveniência, sendo que as } \\
\text { dimensões estrutura } \\
\text { comunicação não foram } \\
\text { significativas na satisfação dos } \\
\text { usuários do plano de saúde. }\end{array}$ \\
\hline
\end{tabular}

Fonte: Elaborado pelos autores.

Como pode ser observado na Tabela 1 , os estudos buscam encontrar dimensões que auxiliam na avaliação da qualidade dos serviços de saúde prestados, seja validando instrumentos existentes no exterior, seja avaliando a qualidade do serviço em si.

\subsection{Análise de Importância e Desempenho}

Um dos métodos utilizados para identificação do que deveria ser melhorado, em produtos e serviços, é a análise de importância x desempenho. Basicamente, esta análise considera a percepção dos usuários em relação à importância dos aspectos relevantes de um determinado serviço e do desempenho do estabelecimento que presta o serviço (MIRANDA et al., 2010). A análise de importância e desempenho (Importance-Performance Analysis - IPA) tem sido utilizada como uma ferramenta para avaliar a posição competitiva da empresa no mercado, visando identificar oportunidades de melhoria e para orientar os esforços de planejamento estratégico (GARVER, 2003). 
A IPA foi originalmente proposta por Martilla e James (1977), como uma ferramenta gráfica simples para promover o desenvolvimento de estratégias de marketing eficazes, baseadas em juízos sobre a importância e o desempenho de cada atributo. Tipicamente, os dados provenientes de pesquisas de satisfação dos clientes são usados para avaliar prioridades de melhoria. Atributos cujos usuários do serviço dão alta importância, mas que têm alto desempenho, representam pontos fortes. Um atributo de alta importância, mas de baixo desempenho, deve receber atenção imediata. Atributos com baixa importância e baixo desempenho, não requerem esforço adicional sobre eles. Para atributos com alto desempenho, mas com baixa importância, a empresa pode estar desperdiçando recursos que poderiam ser melhor utilizados em outro lugar. Uma possível desvantagem desta abordagem de quadrantes é que "uma pequena alteração na posição de um atributo pode levar a uma mudança dramática na prioridade inferida do atributo" (ESKILDSEN; KRISTENSEN, 2006, p.42). Devido a limitações da IPA original (ESKILDSEN; KRISTENSEN, 2006), Slack (1994) propõe uma análise assimétrica, identificando a prioridade de melhorias por meio da diferença entre a importância dada pelos usuários do serviço, a cada aspecto pesquisado, e o desempenho de cada um desses aspectos sob o ponto de vista dos usuários (eq. 1).

\section{Prioridade $=$ Importância - Desempenho $\quad($ Eq.1)}

A abordagem de Slack permite uma transição mais contínua nas prioridades inferidas (ESKILDSEN; KRISTENSEN, 2006) e o raciocínio por trás disso é que os clientes podem aceitar um desempenho menor em atributos menos importantes e exigem maior desempenho de atributos mais importantes.

A avaliação do desempenho dos atributos no IPA normalmente é efetuada através da utilização de escalas de desempenho (satisfação dos usuários com o serviço prestado) (GARVER, 2003). Por outro lado, duas maneiras podem ser usadas para estimar a importância dos atributos: importância declarada ou importância estatisticamente inferida. No método de importância declarada, os clientes são solicitados a classificar a importância do atributo variando tipicamente de "sem importância" a "muito importante", em uma escala de Likert. Este método apresenta algumas limitações: Os consumidores tendem a dar maior importância aos atributos que representam as funções básicas de um serviço (GARVER, 2003); e, geralmente, a importância declarada tende a ter baixo poder de discriminação e os clientes tendem a dizer tudo como importante. Isso ocorre porque os clientes estão argumentando sobre a importância dos atributos, o que pode resultar em respostas socialmente aceitáveis ou status quo (GUSTAFSSON; JOHNSON, 2004).

Para a importância estatisticamente inferida, os clientes são convidados a avaliar tanto a sua satisfação com o desempenho atual dos diferentes atributos, quanto sua satisfação geral com o serviço em estudo. Vários métodos podem ser usados para identificar a importância, tais como: correlação linear, regressão múltipla, estimativa de pares normalizados, etc. (GUSTAFSSON; JOHNSON, 2004). Neste artigo, usamos a análise de regressão entre a satisfação com os aspectos pesquisados, e a avaliação geral do serviço, sob o ponto de vista dos usuários. Quando o desempenho é avaliado pela satisfação dos usuários, e a importância é estimada pela regressão linear entre a satisfação com os aspectos pesquisados e a satisfação geral com o serviço (método de importância inferida).

Tanto a satisfação quanto a importância de cada característica são padronizadas segundo a fórmula:

$$
\operatorname{StdVar}_{i}=\frac{\operatorname{Var}_{i}-\overline{\operatorname{Var}}}{\operatorname{DevPad}_{\operatorname{Var}}}
$$


Onde

$\operatorname{Var}_{i}=$ Satisfação (ou importância) dos usuários com variável "i” avaliada (Ex: atendimento, comunicação, etc.).

StdVar ${ }_{i}=$ Variável "i" padronizada

$\overline{V a r}=$ Média de todas as variáveis analisadas

DevPad $_{\text {Var }}=$ Desvio padrão das variáveis analisadas

\section{METODOLOGIA}

O presente estudo apresenta quanto aos objetivos, uma pesquisa do tipo exploratória. Quanto aos procedimentos, levantamento. E quanto a abordagem, quantitativa (HAIR et al, 2009).

O instrumento de coleta de dados caracteriza-se por um questionário com duas partes. $\mathrm{Na}$ primeira parte pediu-se aos respondentes para assinalarem sua satisfação ou insatisfação quanto as dimensões atendimento, comunicação, instalações, acesso, tempo, disponibilidade de serviços, farmácia, segurança e desempenho. Na última etapa perguntaram-se questões pessoais, quanto idade, gênero, frequência de uso, etc., bem como sua satisfação geral com os serviços que acessam.

A aplicação do questionário foi realizada in loco, nos 7 Ambulatórios Gerais de Blumenau/SC entre julho e agosto de 2016. Obteve-se o total de 355 questionários respondidos, sendo uma amostragem não probabilística, aplicada de forma aleatória. Foi utilizado um questionário com 39 variáveis cuja resposta variava em uma Escala Lickert correspondente entre 1 e 5, totalmente insatisfeito e totalmente satisfeito, respectivamente. Utilizou-se análise de Importância x Desempenho (MARTILLA; JAMES, 1977; SLACK, 1994). Os dados foram importados para uma planilha do Software Microsoft Excel e depois para o Software Estatístico SPSS® (Statistical Package for the Social Sciences), versão 22, e foi realizada a análise de Regressão Linear.

Para analisar os possíveis atributos e dimensões que interferem na matriz de importância e desempenho em relação a ambulatórios gerais, foi realizada pesquisa na literatura sobre o tema. Com isso, foram encontrados e elaborados os seguintes atributos e dimensões conforme a Tabela 2:

Tabela 2: Dimensões da pesquisa.

\begin{tabular}{|c|c|c|}
\hline Dimensão & Descrição & Autores \\
\hline Atendimento & $\begin{array}{l}\text { Aborda o respeito e simpatia dos atendentes durante } \\
\text { o atendimento; Vontade dos atendentes em lhe } \\
\text { auxiliar, entendendo o que os clientes precisam. }\end{array}$ & $\begin{array}{l}\text { Barbosa et al, (2009), Cho et al, } \\
\text { (2004), Coulter, Fitzpatrick e } \\
\text { Cornwell, (2009), Fadel e Regis Filho, } \\
\text { (2009), Gasquet et al, (2004), Milan e } \\
\text { Trez, (2005), Soufi et al (2010), } \\
\text { Padma, Rajendran e Sai, (2009). }\end{array}$ \\
\hline Comunicação & $\begin{array}{l}\text { Concordância de informações e orientações dadas } \\
\text { por diferentes equipes do Ambulatório; Clareza e } \\
\text { suficiência das informações, além do fornecimento } \\
\text { de informações solicitadas. }\end{array}$ & $\begin{array}{l}\text { Padma, Rajendran e Sai, (2009), } \\
\text { Fadel e Regis Filho, (2009). }\end{array}$ \\
\hline $\begin{array}{l}\text { Qualidade das } \\
\text { instalações }\end{array}$ & $\begin{array}{l}\text { Aborda questões do conforto e aparência das } \\
\text { instalações, adequação dos equipamentos e }\end{array}$ & $\begin{array}{l}\text { Barbosa et al, (2009), Cho et al, } \\
\text { (2004), Coulter, Fitzpatrick e } \\
\text { Cornwell, (2009), Fadel e Regis Filho, }\end{array}$ \\
\hline
\end{tabular}




\begin{tabular}{|c|c|c|}
\hline & $\begin{array}{l}\text { instalações aos serviços prestados. Limpeza e } \\
\text { higiene geral do ambiente. }\end{array}$ & $\begin{array}{l}\text { (2009), Gasquet et al, (2004), Milan e } \\
\text { Trez, (2005), Wei et al, (2015), } \\
\text { Padma, Rajendran e Sai, (2009). }\end{array}$ \\
\hline $\begin{array}{c}\text { Acesso ao } \\
\text { Ambulatório Geral }\end{array}$ & Localização e facilidade de acesso ao ambulatório. & $\begin{array}{l}\text { Barbosa et al, (2009), Fadel e Regis } \\
\text { Filho, (2009), Milan e Trez, (2005). }\end{array}$ \\
\hline $\begin{array}{l}\text { Tempo/Prontidão } \\
\text { atendimento }\end{array}$ & $\begin{array}{l}\text { Horários de funcionamento e agendamento } \\
\text { disponíveis no ambulatório; Pontualidade e tempo } \\
\text { de espera nas consultas agendadas; Duração das } \\
\text { consultas pelos profissionais de saúde; Tempo entre } \\
\text { a solicitação da consulta e sua realização. }\end{array}$ & $\begin{array}{l}\text { Barbosa et al, (2009), Cho et al, } \\
\text { (2004), Coulter, Fitzpatrick e } \\
\text { Cornwell, (2009), Fadel e Regis Filho, } \\
\text { (2009), Gasquet et al, (2004), Milan e } \\
\text { Trez, (2005), Padma, Rajendran e Sai, } \\
\text { (2009), Soufi et al (2010), Wei et al, } \\
(2015) \text {. }\end{array}$ \\
\hline $\begin{array}{l}\text { Disponibilidade de } \\
\text { serviço }\end{array}$ & $\begin{array}{l}\text { Especialidades disponíveis para atendimento no } \\
\text { ambulatório, além do serviço médico (pediatra, } \\
\text { ginecologista, clínico geral.). }\end{array}$ & Barbosa et al, (2009). \\
\hline $\begin{array}{l}\text { Farmácia/ } \\
\text { medicamentos }\end{array}$ & $\begin{array}{l}\text { Disponibilidade dos medicamentos que você } \\
\text { necessita e tempo até conseguir na farmácia do } \\
\text { ambulatório, a medicação necessária. }\end{array}$ & $\begin{array}{l}\text { Barbosa et al, (2009), Milan; Trez, } \\
\text { (2005). }\end{array}$ \\
\hline $\begin{array}{l}\text { Confidencialidade e } \\
\text { confiabilidade dos } \\
\text { serviços prestados }\end{array}$ & $\begin{array}{l}\text { Privacidade, identificação e atendimento pessoal } \\
\text { durante o atendimento dos pacientes; Percepção de } \\
\text { segurança quanto a prevenção de infecção no } \\
\text { ambulatório. }\end{array}$ & $\begin{array}{l}\text { Barbosa et al, (2009), Coulter, } \\
\text { Fitzpatrick e Cornwell, (2009), } \\
\text { Duggirala et al (2008), Padma, } \\
\text { Rajendran e Sai, (2009), Soufi et al } \\
\text { (2010), Wei et al, (2015). }\end{array}$ \\
\hline $\begin{array}{l}\text { Desempenho / } \\
\text { Resultados } \\
\text { conseguidos no } \\
\text { Ambulatório Geral }\end{array}$ & 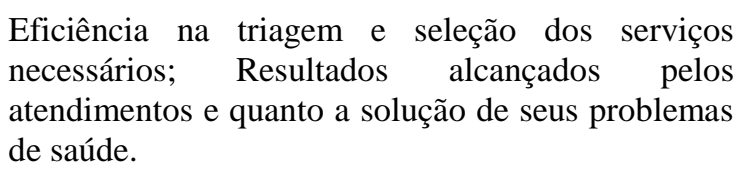 & Duggirala et al, (2008). \\
\hline
\end{tabular}

Fonte: Elaborado pelos autores.

As dimensões e variáveis expostas na Tabela 2 foram analisadas por meio da Análise Fatorial com rotação Varimax, com vistas a verificar sua validade e confiabilidade, conforme disposto na tabela 3. Verifica-se que as variáveis mensuradas se agruparam nas nove dimensões pesquisadas, tendo 74\% de variância extraída, Kaiser-Meyer-Olkin $(\mathrm{KMO})=0,852$ e Alfa de Cronbach superior a 0,7 .

Tabela 3 - Matriz de componente rotativa ${ }^{a}$

\begin{tabular}{|c|c|c|c|c|c|c|c|c|c|c|c|}
\hline & 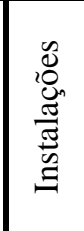 & 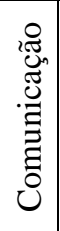 & 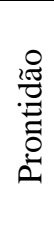 & 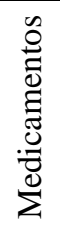 & 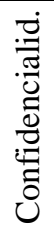 & 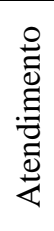 & 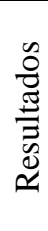 & 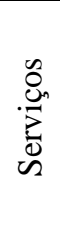 & 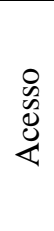 & $\begin{array}{l}\sum_{0}^{\pi} \\
\sum_{\Sigma}^{\mathbb{N}}\end{array}$ & 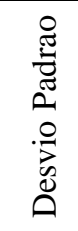 \\
\hline $\begin{array}{l}\text { Inst1 - Aparência física do Ambulatório Geral } \\
\text { Inst2 - Conservação do Ambulatório Geral } \\
\text { Inst3 - Conservação de equipamentos médicos } \\
\text { Inst4 - Iluminação do ambulatório em geral } \\
\text { Inst5 - Limpeza e higiene geral do ambiente }\end{array}$ & $\begin{array}{l}0.85 \\
0.83 \\
0.82 \\
0.78 \\
0.74\end{array}$ & & & & & & & & & $\begin{array}{l}4.02 \\
4.06 \\
4.02 \\
4.40 \\
4.32\end{array}$ & $\begin{array}{l}1.12 \\
1.07 \\
1.04 \\
0.86 \\
0.88\end{array}$ \\
\hline
\end{tabular}




\begin{tabular}{|c|c|c|c|c|c|c|c|c|c|c|c|}
\hline Inst6 - Climatização (Ar condicionado) & 0.72 & & & & & & & & & 4.28 & 1.01 \\
\hline Inst7 - Local para sentar enquanto aguarda atendimento & 0.71 & & & & & & & & & 4.07 & 1.13 \\
\hline Inst8 - Facilidade de acesso do Ambulatório Geral & 0.68 & & & & & & & & & 4.34 & 0.92 \\
\hline Inst9 - Sinalização/Orientação interna do Ambulatório Geral & 0.65 & & & & & & & & & 4.14 & 1.03 \\
\hline Inst10 - Disponibilidade de equipamentos médicos necessários & 0.61 & & & & & & & & & 4.07 & 0.94 \\
\hline Com1- Quão suficientes são informações fornecidas & & 0.77 & & & & & & & & 4.05 & 0.97 \\
\hline Com2 - Clareza das informações (orientações) prestadas & & 0.72 & & & & & & & & 4.11 & 0.98 \\
\hline Com3 - Concordância informações entre equipes do Ambul. & & 0.71 & & & & & & & & 3.93 & 1.04 \\
\hline Com4 - Fornecimento de informações que você solicita & & 0.70 & & & & & & & & 4.13 & 0.97 \\
\hline Prontidão 1 - Tempo de espera para ser atendido & & & 0.81 & & & & & & & 3.57 & 1.13 \\
\hline Prontidão 2 - Pontualidade nas consultas agendadas & & & 0.72 & & & & & & & 3.54 & 1.14 \\
\hline Prontidão 3 - Tempo entre solicitação e realização da consulta & & & 0.67 & & & & & & & 3.48 & 1.16 \\
\hline Prontidão 4 - Horários disponíveis para agendamento & & & 0.63 & & & & & & & 3.19 & 1.16 \\
\hline Medicam 1 - Disponibilidade da medicação receitada & & & & 0.91 & & & & & & 3.35 & 1.17 \\
\hline Medicam 2 - Disponibilidade medicamentos que você necessita & & & & 0.89 & & & & & & 3.33 & 1.20 \\
\hline Medicam 3 - Tempo conseguir na farmácia a medicação & & & & 0.86 & & & & & & 3.34 & 1.24 \\
\hline Confidencialid. 1 -Identificação pessoal durante o atendimento & & & & & 0.72 & & & & & 4.27 & 0.98 \\
\hline Confidencialid. 2- Privacidade durante o atendimento & & & & & 0.72 & & & & & 4.40 & 0.89 \\
\hline Confidencialid. 3 -Prevenção de infecção no ambulatório & & & & & 0.63 & & & & & 4.11 & 0.99 \\
\hline Confidencialid. 4 -Acesso do médico ao seu histórico de saúde & & & & & 0.60 & & & & & 4.28 & 1.01 \\
\hline Atendimento 1 - Respeito atendimento & & & & & & 0.79 & & & & 4.04 & 1.05 \\
\hline Atendimento 2 - Vontade dos atendentes em lhe auxiliar & & & & & & 0.77 & & & & 3.70 & 1.19 \\
\hline Atendimento 3 - Simpatia atendentes no contato com usuários & & & & & & 0.76 & & & & 3.76 & 1.15 \\
\hline Result3 - Resultados alcançados nos atendimentos realizados & & & & & & & 0.76 & & & 3.96 & 0.92 \\
\hline Result4 - Solução de seus problemas de saúde & & & & & & & 0.73 & & & 3.91 & 0.94 \\
\hline Result2 - Capacidade Ambulatório identificar suas necessidades & & & & & & & 0.69 & & & 3.92 & 0.94 \\
\hline Serv Dispon 1 - Especialidades disponíveis para atendimento & & & & & & & & 0.79 & & 3.47 & 1.14 \\
\hline Serv Dispon 3 -Variedade serv. disponíveis, além de médicos & & & & & & & & 0.73 & & 3.49 & 1.13 \\
\hline Serv Dispon 2 -Disponibilidade profissional de sua preferência & & & & & & & & 0.66 & & 3.41 & 1.17 \\
\hline Acesso 1 - Localização do ambulatório & & & & & & & & & 0.86 & 4.36 & 0.95 \\
\hline Acesso 3 - Facilidade para acesso (Ônibus, táxis, etc.) & & & & & & & & & 0.85 & 4.29 & 1.03 \\
\hline Alfa Cronbach & & & 0.83 & 0.91 & 0.82 & 0.88 & & 0.81 & 0.78 & & \\
\hline \% Variância extraída & 18.4 & 27.6 & 35.6 & 43.2 & 50.2 & 56.9 & 63.1 & 69.1 & 74.0 & & \\
\hline
\end{tabular}

Fonte: Dados da pesquisa.

De acordo com Marôco (2011) o KMO é uma medida da homogeneidade das variáveis, que compara as correlações simples com as correlações parciais observadas entre as variáveis. Conforme indicado por Hair et al (2005) o resultado acima de 0,800 é considerado bom, entre 0,700 e 0,600 é considerado razoável, entre 0,600 e 0,500 considerado um resultado ruim, e abaixo de 0,500 considerado inaceitável. O Alpha de Crombach tem por finalidade a análise da confiabilidade no que tange as variáveis agrupadas dentro de cada dimensão, sendo que acima de 0,7 é considerado bom.

A variável dependente é representada pela dimensão Desempenho, que é composta pela média de concordância com as seguintes afirmações: Geral1 - Os serviços prestados por este ambulatório têm qualidade; Geral2 - Este ambulatório atende aquilo que deveria fazer; Geral3 - Os serviços deste ambulatório atendem minhas necessidades; Geral4 - Estou satisfeito com este Ambulatório Geral; Geral5 - Recomendaria este ambulatório a quem me perguntasse.

A dimensão de desempenho dos serviços prestados rotacionou em apenas uma dimensão, explicando 78,03\% da variância dos fatores que a compõem, com Alfa de Cronbach 0,929 e teste de Kaiser-Meyer-Olkin $(\mathrm{KMO})=0,887$.

\section{APRESENTAÇÃO E ANÁLISE DOS RESULTADOS}


Nesta sessão serão apresentados o perfil da amostra da pesquisa e o resultado da Análise de Importância e Desempenho das dimensões utilizadas no estudo para avaliar a avaliar a qualidade dos serviços em saúde pública nos Ambulatórios Gerais de Blumenau, assim como na identificação das melhorias no serviço. A Tabela 4 apresenta os dados relativos ao perfil dos respondentes da pesquisa.

Tabela 4: Perfil da amostra.

\begin{tabular}{l|r|l|r|l|l|l|r}
\multicolumn{2}{c|}{ Gênero } & \multicolumn{2}{c|}{ Escolaridade } & Renda familiar & \multicolumn{3}{c}{ Idade } \\
\hline Masculino & $60,5 \%$ & Até 4 série & $14,9 \%$ & 2 ou menos & $46 \%$ & Até 20 anos & $8,9 \%$ \\
\hline Feminino & $37,1 \%$ & Da 5 a $8^{\circ}$ série & $22,2 \%$ & 3 a 5 & $42 \%$ & 21 a 30 & $22,40 \%$ \\
\hline Ausente & $2,3 \%$ & Ensino médio & $43,6 \%$ & Mais que 5 & $7,3 \%$ & 31 a 40 & $24,70 \%$ \\
\hline & & Superior & $16,6 \%$ & Ausente & $5,1 \%$ & 41 a 50 & $19,30 \%$ \\
\hline & & Não sei & $0,5 \%$ & & & Acima de 50 & $24,70 \%$ \\
\hline & & Ausente & $2 \%$ & & & Ausente & $0.2 \%$ \\
\hline
\end{tabular}

Fonte: dados da pesquisa.

Verifica-se que o perfil predominante dos respondentes destaca-se como sendo do gênero masculino, com ensino médio e que recebe menos que dois salários mínimos ( $\mathrm{R} \$$ 880,00). A idade apresentou um resultado heterogêneo, sem predominância de uma faixa etária específica.

Os dados foram coletados no seguintes Ambulatórios Gerais na cidade de Blumenau: Velha, Itoupava Central, Garcia, Centro, Fortaleza, Badenfurt e Escola Agrícola. A Tabela 5 apresenta o percentual de amostra coletada em cada ambulatório. Como observado, o percentual de respondentes em cada Ambulatório foi homogêneo e alcançou todos os Ambulatórios Gerais presentes na cidade de Blumenau.

Tabela 5: Ambulatórios Gerais de Blumenau.

\begin{tabular}{c|c|c|c|c|c|c}
\hline Velha & $\begin{array}{c}\text { Itoupava } \\
\text { Central }\end{array}$ & Garcia & Centro & Fortaleza & Badenfurt & $\begin{array}{c}\text { Escola } \\
\text { Agrícola }\end{array}$ \\
\hline $15,5 \%$ & $13,8 \%$ & $14,4 \%$ & $14,9 \%$ & $13,5 \%$ & $14,1 \%$ & $13,8 \%$ \\
\hline
\end{tabular}

Fonte: dados da pesquisa.

A Tabela 6 mostra o resultado da análise de regressão linear entre a satisfação dos usuários dos serviços de saúde prestados pelos Ambulatórios, em relação a diferentes dimensões, e a avaliação geral que fazem sobre o desempenho dos serviços prestados.

Tabela 6 - Resultados Equação de Regressão Linear

\begin{tabular}{|ll|c|c|c|c|c|}
\hline \multirow{2}{*}{} & \multicolumn{2}{|c|}{$\begin{array}{c}\text { Coeficientes não } \\
\text { padronizados }\end{array}$} & $\begin{array}{c}\text { Coeficientes } \\
\text { padroniz. }\end{array}$ & & \\
\cline { 2 - 5 } & \multicolumn{2}{|c|}{$\begin{array}{c}\text { Modelo } \\
\text { padrão }\end{array}$} & Beta & $\mathrm{t}$ & Sig. \\
\hline & (Constante) & .003 & .161 & & -.016 & .987 \\
1 & Resultados & .303 & .043 & .318 & 7.111 & .000 \\
2 & Confidencialidade & .223 & .047 & .228 & 4.787 & .000 \\
3 & Atendimento & .186 & .035 & .226 & 5.387 & .000 \\
4 & Serviços Disponíveis & .094 & .033 & .109 & 2.853 & .005 \\
5 & Instalações & .089 & .043 & .085 & 2.065 & .040 \\
6 & Medicamento & .079 & .025 & .104 & 3.154 & .002 \\
7 & Prontidão & .071 & .035 & .078 & 1.997 & .047
\end{tabular}




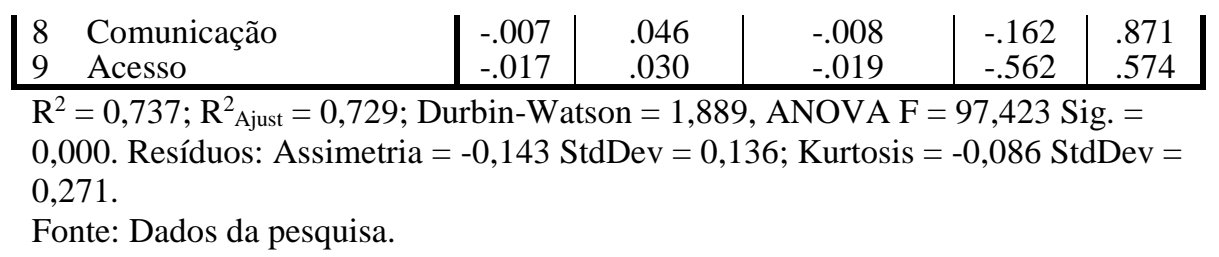

Os resultados demonstram que as dimensões pesquisadas explicam $72,9 \%\left(\mathrm{R}^{2}\right.$ Ajust $=$ 0,729 ) da variância da avaliação geral dos serviços prestados e os resíduos seguem distribuição normal (Assimetria e Kurtosis < 2* StdDev; Durbin-Watson =1,889). No geral, a equação resultante é significativa (ANOVA F $=97,423 \mathrm{Sig}=0,000$ ).

Entre as dimensões pesquisadas, a mais impactante na avaliação geral diz respeito aos resultados (em termos de saúde) conseguidos pelos serviços prestados $(B=0,303)$. Na sequência estão a Confidencialidade $(B=0,223)$ e o Atendimento $(B=0,186)$. Já o Acesso aos ambulatórios (localização e transporte público) e a Comunicação não tiveram impacto na avaliação geral dos serviços prestados ( $\mathrm{p}$-value > 0,05).

A tabela 7 mostra o nível de desempenho atual das dimensões estudadas, bem como a análise da lacuna de importância - desempenho, permitindo a priorização das ações de melhoria.

Tabela 7 - Resultados Análise Importância x Desempenho

\begin{tabular}{|c|c|c|c|c|c|c|c|c|c|}
\hline & 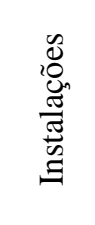 & 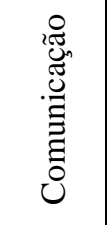 & 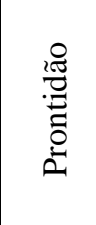 & 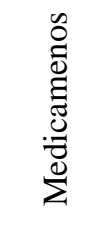 & 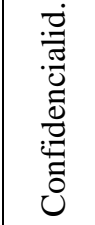 & 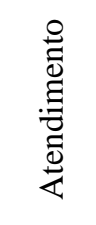 & 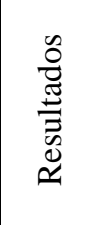 & 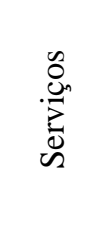 & 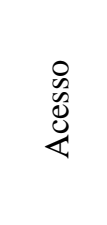 \\
\hline Média Dimensão & 4.17 & 4.05 & 3.44 & 3.35 & 4.26 & 3.84 & 3.91 & 3.46 & 4.31 \\
\hline Desvio Padrão Dimensão & 0.79 & 0.86 & 0.92 & 1.10 & 0.85 & 1.01 & 0.87 & 0.83 & 0.91 \\
\hline $\begin{array}{l}\text { Coeficiente Regressão } \\
\text { (Importância inferida) }\end{array}$ & 0,089 & $\approx 0,00$ & 0,071 & 0,079 & 0,223 & 0,186 & 0,303 & 0,094 & $\approx 0,00$ \\
\hline Std Desempenho & 0,83 & 0,49 & $-1,15$ & $-1,39$ & 1,06 & $-0,06$ & 0,12 & $-1,11$ & 1,20 \\
\hline Std Importância & $-0,27$ & $-1,14$ & $-0,44$ & $-0,36$ & 1,05 & 0,69 & 1,84 & $-0,22$ & $-1,14$ \\
\hline $\begin{array}{l}\text { Std Importância - Std } \\
\text { Desempenho }\end{array}$ & $-1,1$ & $-1,63$ & 0,71 & 1,03 & $-0,01$ & 0,75 & 1,72 & 0,89 & $-2,34$ \\
\hline Prioridade para melhoria & 7 & 8 & 5 & 2 & 6 & 4 & 1 & 3 & 9 \\
\hline $\begin{array}{l}\text { \% Acima ou abaixo da } \\
\text { avaliação geral }\end{array}$ & $4 \%$ & $1 \%$ & $-13 \%$ & $-15 \%$ & $7 \%$ & $-3 \%$ & $-2 \%$ & $-13 \%$ & $8 \%$ \\
\hline
\end{tabular}

Fonte: Dados da pesquisa.

Por meio da Tabela 7 pode-se verificar a satisfação que os respondentes apresentaram no que tange as dimensões estudadas. Verifica-se que tiveram desempenho superior $(>4)$ apenas as dimensões Instalações, Comunicação, Confidencialidade e Acesso. Já as dimensões relacionadas aos Serviços complementares disponíveis, disponibilidade de medicamentos, prontidão no atendimento, atendimento e resultados em termos de saúde se mantiveram entre 3 e 4, em uma escala utilizada de 1 a 5 . Os resultados relativos ao desvio padrão, caracterizado pela dispersão dos dados em relação à média, foi mais baixo na dimensão instalações, demonstrando homogeneidade das instalações (menor variabilidade na avaliação dos usuários de diferentes ambulatórios). Já a dimensão disponibilidade de medicamentos e atendimento apresentaram alta dispersão, representando heterogeneidade dos dados.

Importante salientar que a avaliação geral dos serviços prestados teve uma média de 3,99 e Desvio Padrão de 0,83. Realizando um teste t-pareado, verifica-se que não há diferença 
significativa ( $\mathrm{p}$-value > 0,05) apenas entre a avaliação geral e a dimensão comunicação. Quanto as outras, verifica-se que os respondentes têm uma percepção melhor que a avaliação geral em relação as seguintes dimensões: Instalações (Diferença $=-0,180 ; \mathrm{p}$-value $=0,000$ ), Confidencialidade / segurança (Diferença $=-0,269 ; \mathrm{p}$-value $=0,000)$, Acesso $($ Diferença $=-$ $0,324 ; \mathrm{p}$-value $=0,000)$. Já os clientes têm uma avaliação inferior à avaliação geral quanto as seguintes dimensões: Disponibilidade de medicamentos (Diferença $=0,647 ; \mathrm{p}$-value $=0,000$ ), Prontidão no atendimento (Diferença $=0,543 ; \mathrm{p}$-value $=0,000$ ), Serviços complementares $($ Diferença $=0,533 ; \mathrm{p}$-value $=0,000)$, Atendimento $($ Diferença $=0,154 ; \mathrm{p}$-value $=0,001)$, Resultados (Diferença $=0,073 ; \mathrm{p}$-value $=0,028$ ).

Analisando apenas as diferenças, verifica-se que a Disponibilidade de medicamentos, a Prontidão no atendimento e os Serviços complementares seriam as dimensões com prioridade de melhoria (mais baixos desempenho em relação a avaliação geral). Porém, verificando o impacto destas dimensões na avaliação geral dos serviços (tabela 6), verifica-se que, embora a disponibilidade de medicamentos tenha a maior diferença na média da satisfação (Diferença = 0,647; p-value $=0,000$ ), ela tem o terceiro menor impacto. Este resultado demonstra a relevância de não apenas considerar o desempenho das diferentes dimensões avaliadas, mas também a relevância de cada uma.

Deste modo, realizando a análise da lacuna entre a importância e o desempenho, seguindo o proposto por Slack (1994) para determinação das prioridades de melhoria (Tabela 7), verifica-se que a sequência de prioridades para melhoria é: 1 - Resultados obtidos, seguido por 2 - Disponibilidade de medicamentos, 3 - Serviços complementares, 4 - Atendimento, 5 Prontidão no atendimento, 6 - Confidencialidade, 7 - Instalações, 8 - Comunicação e 9 Acesso.

As ações definidas como prioridade de melhoria podem ser definidas com base nos atributos mensurados em cada dimensão. Por exemplo, em relação a prontidão / rapidez para atendimento, esta dimensão se refere a horários de funcionamento do ambulatório (Média = $3,19)$; pontualidade nas consultas agendadas $(3,54)$; horários disponíveis para agendamento $(3,19)$; tempo entre a solicitação da consulta e sua realização $(3,48)$. Algumas dessas ações podem ser melhoradas somente com a reorganização de agenda, como a pontualidade das consultas agendadas. Outras ações exigem contratação de pessoal para atendimento ocasionando em um aumento substancial dos gastos públicos. Assim, é possível analisar cada uma das dimensões e suas prioridades de melhoria para identificar e encontrar uma melhor maneira de aprimorar os serviços oferecidos. Cabe ao gestor estudar e avaliar a melhor estratégia a ser implantada visando uma maior qualidade e satisfação dos serviços de saúde pública ofertados aos cidadãos usuários.

\section{CONSIDERAÇÕES FINAIS}

O estudo teve como objetivo avaliar a qualidade dos serviços em saúde pública em Ambulatórios Gerais na cidade de Blumenau. E além disso, identificar melhorias que podem ser realizadas no serviço ofertado aos usuários.

Como resultados verificou-se que os usuários se encontram, de modo geral, satisfeitos no que tange as dimensões Instalações, Confidencialidade / segurança e Acesso, tendo um desempenho superior a avaliação geral que fazem dos Ambulatórios Gerais de Saúde na cidade de Blumenau / SC. Já os usuários estão menos satisfeitos com Disponibilidade de medicamentos, Prontidão no atendimento, Serviços complementares, Atendimento e Resultados. 
No tocante a importância, a dimensão Resultados obteve a maior relevância, seguida por Confidencialidade, Atendimento, Serviços Disponíveis, Instalações, Medicamentos, Prontidão, Comunicação e Acesso.

Entretanto, não basta identificar as dimensões com maior importância ou desempenho realizados nos serviços públicos de saúde. É necessário avaliar os pontos de melhoria de modo a contribuir para ações estratégicas que visem o aumento de qualidade e satisfação nos serviços ofertados aos usuários. O método de Análise de Importância x Desempenho de Slack (1994) vem a contribuir neste sentido. De modo que, pôde-se identificar, na avaliação dos usuários dos serviços de saúde pública ofertados em Ambulatórios Gerais de Blumenau, as seguintes prioridades de melhoria: 1 - Resultados obtidos, seguido por 2 - Disponibilidade de medicamentos, 3 - Serviços complementares, 4 - Atendimento, 5 - Prontidão no atendimento, 6 - Confidencialidade, 7 - Instalações, 8 - Comunicação e 9 - Acesso.

Espera-se com este estudo contribuir para a reflexão e futuras ações que visem melhorar os serviços prestados aos usuários dos serviços em saúde pública. Pois mesmo os usuários mostrando-se satisfeitos em relação aos serviços, o mesmo pode ter sua qualidade melhorada levando a maior satisfação. Buscando converter em ações estratégicas que auxiliem na tomada de decisão e subsidiem aperfeiçoamentos no âmbito dos serviços (Esperidião e Trad, 2006), os resultados da presente pesquisa são relevantes, na medida em que, conforme afirmam Giovanella et al (2013), podem contribuir para a institucionalização da avaliação e reorganização dos serviços, além de ser uma ferramenta para a participação popular.

Quanto as limitações do presente estudo, destaca-se o fato de ter sido realizado em um município na região sul, especificamente. Deste modo, seus achados não podem ser generalizados no tocante aos serviços de saúde pública no país. Além disso, estudos qualitativos seriam relevantes na identificação de questões mais profundas visto que a saúde pública, apesar de seus usuários se mostrarem satisfeitos de modo geral como constatado no presente estudo, há muito para ser melhorado em relação a qualidade na área dos serviços públicos de saúde.

\section{REFERÊNCIAS}

ANSUJ, A. P.; ZENCKNER, C. L.; GODOY, L. P. Percepção da qualidade dos serviços de odontologia. In: ENCONTRO NACIONAL DE ENGENHARIA DE PRODUÇÃO, v. 25, 2005, Porto Alegre.

BARBOSA, A. C. Q.; RODRIGUES, J. M.; SILVA, N. C.; ROCHA, T. A. H. Proposição de metodologia de avaliação de desempenho das Equipes de Saúde da Família e satisfação dos usuários: um desenho elaborado a partir das cidades de Minas Gerais. Belo Horizonte: Observatório de Recursos Humanos em Saúde, 2009.

BOLTON, R. N.; DREW, J. H. A multistage model of customers assessment of service quality and value. Journal of Consumer Research, v. 17, n. 4, p. 375-84, 1991.

CHO, W. H.; LEE, H.; KIM, C.; LEE, S.; CHOI, K. The impact of visit frequency on the relationship between service quality and outpatient satisfaction: a South Korean study. Health services research, v. 39, n. 1, p. 13-34, 2004.

COULTER, A.; FITZPATRICK, R.; CORNWELL, J. Measures of patients' experience in hospital: purpose, methods and uses. London: King's Fund, 2009.

CRONIN, J. J.; TAYLOR, S. A. Measuring service quality: a reexamination and extension. Journal of Marketing, v.56, n.3, p.55-68, jul 1992. 
DINSDALE G. B.; MANSON D. B.; SCHMIDT F.; STRICKLAND T. Metodologia para medir a satisfação do usuário no Canadá: desfazendo mitos e redesenhando roteiros. Brasília, Escola Nacional de Administração Pública, ENAP, Nº 20, 2000.

DUGGIRALA, M.; RAJENDRAN, C.; ANANTHARAMAN, R. N. Patient-perceived dimensions of total quality service in healthcare. Benchmarking: An International Journal, v. 15, n. 5, p. 560-583, 2008.

ESPERIDIÃO, M. A.; TRAD, L. A. B. Avaliação de satisfação de usuários: considerações teórico-conceituais User satisfaction assessment: theoretical and conceptual concerns. Cad. Saúde Pública, v. 22, n. 6, p. 1267-1276, 2006.

ESKILDSEN, J. K.; KRISTENSEN, K. Enhancing importance-performance analysis. International Journal of Productivity and Performance Management, v. 55, n. 1, p. 40-60, 2006.

FADEL, M. A. V.; REGIS FILHO, G. I. Percepção da qualidade em serviços públicos de saúde: um estudo de caso. Revista de Administração Pública, v. 43, n. 1, p. 7-22, 2009.

FRÉZ, A. R.; NOBRE, M. I. R. de S. Satisfação dos usuários dos serviços ambulatoriais de fisioterapia da rede pública. Fisioter Mov, v. 24, n. 3, p. 419-28, 2011.

GARVER, M. S. Best practices in identifying customer-driven improvement opportunities. Industrial Marketing Management, v. 32, n. 6, p. 455-466, 2003.

GASQUET, I.; VILLEMINOT, S.; ESTAQUIO, C.; DURIEUX, P.; RAVAUD, P.; FALISSARD, B. Construction of a questionnaire measuring outpatients' opinion of quality of hospital consultation departments. Health and quality of life outcomes, v. 2, n. 1, p. 1, 2004.

BRANDÃO, A. L. R. B. S.; GIOVANELlA, L.; CAMPOS, Carlos Eduardo Aguilera. Avaliação da atenção básica pela perspectiva dos usuários: adaptação do instrumento EUROPEP para grandes centros urbanos brasileiros. Ciência \& Saúde Coletiva, v. 18, n. 1, p. 103-114, 2013.

GRÖNROOS, C. A service quality model and its marketing implications. European Journal of Marketing, v. 18, n. 4, p. 36-44, 1984.

GUSTAFSSON, A.; JOHNSON, M. D. Determining attribute importance in a service satisfaction model. Journal of Service Research, v. 7, n. 2, p. 124-141, 2004.

HAIR, J. F. et al. Análise multivariada de dados. Bookman Editora, 2009.

LEE, S. M.; LEE, D.; KANG, C. The impact of high-performance work systems in the healthcare industry: employee reactions, service quality, customer satisfaction, and customer loyalty. The Service Industries Journal, Sevilla, 2012.

MALIK, S. U. Customer satisfaction, perceived service quality and mediating role of perceived value. International journal of marketing studies, v. 4 n. 1, fev 2012.

MARTILLA, J. A.; JAMES, J. C. Importance-performance analysis. Journal of Marketing, New York, v, 41, n. 1, p.77 - 79, 1977.

MILAN, G. S.; TREZ, G. Pesquisa de satisfação: um modelo para planos de saúde. RAE eletrônica, v. 4, n. 2, 2005.

MIRANDA, F. J.; CHAMORRO, A.; MURILLO, L. R.; VEGA, Juan. An importanceperformance analysis of primary health care services: managers vs. patients perceptions. J. Service Science \& Management, p. 227-234, jun 2010. 
MOIMAZ, S. A. S.; MARQUES, J. A. M.; SALIBA, O.; GARBIN, C. A. S.; ZINA, L. G.; SALIBA, N. A. Satisfação e percepção do usuário do SUS sobre o serviço público de saúde. Physis: Revista de Saúde Coletiva, p. 1419-1440, 2010.

PADMA, P.; RAJENDRAN, C.; SAI, L. P. A conceptual framework of service quality in healthcare: perspectives of Indian patients and their attendants. Benchmarking: An International Journal, v. 16, n. 2, p. 157-191, 2009.

PARASURAMAN, A.; ZEITHAML, V. A.; BERRY, L. L. A conceptual model of services quality and its implication for future research. Journal of Marketing, v. 49, n. 4, p. 41-50, 1985.

PARASURAMAN, A.; ZEITHAML, V. A.; BERRY, L. L. SERVQUAL: a multiple-item scale for measuring consumer perceptions of service quality. Journal of Marketing, v.64, p.12-40, 1988.

PENA, M. M., et al. O emprego do modelo de qualidade de Parasuraman, Zeithaml e Berry em serviços de saúde. Revista da escola de enfermagem da USP, v. 47 n. 5, oct 2013.

SILVA, L. M. V.; FORMIGLI, V. L A. Avaliação em Saúde: Limites e Perspectivas1. Cad. Saúde Pública, v. 10, n. 1, p. 80-91, 1994.

SLACK, N. The importance-performance matrix as a determinant of improvement priority. International Journal of Operations \& Production Management, v. 14, n. 5, p. 5975, 1994.

SOFTWARE Estatístico SPSS® Statistical Package for the Social Sciences, versão 22.

SOUFI, G.; BELAYACHI, J.; HIMMICH, S.; AHID, S.; SOUFI, M.; ZEKRAOUI, A.; ABOUQAL, R. Patient satisfaction in an acute medicine department in Morocco. BMC health services research, v. 10, n. 1, p. 1, 2010.

TEAS, R.K. Expectations, performance evaluation and consumer's perceptions of quality. Journal of Marketing, v. 57, n. 4, October, p. 18-34, 1993.

VAITSMAN, J.; ANDRADE, G. R. B. Satisfação e responsividade: formas de medir a qualidade e a humanização da assistência à saúde. Ciência e saúde coletiva, v. 10, n. 3, p. 599$613,2005$.

WEI, J.; SHEN, L.; YANG, H. B.; QIN, J. B.; HUANG, W.; ZHANG, J. J.; YANG, T. B. Development and validation of a Chinese outpatient satisfaction questionnaire: evidence from 46 public general hospitals and 5151 outpatients. Public health, v. 129, n. 11, p. 1523-1529, 2015. 